\section{OC-106 THE USE OF INFRARED SPECTROSCOPY TO PROVIDE AN ESTIMATION OF THE GROSS BIOCHEMISTRY ASSOCIATED WITH COLORECTAL PATHOLOGIES}

doi:10.1136/gut.2011.239301.106

J J Wood, ${ }^{1,2^{*}}$ C Kendall, ${ }^{2}$ N Stone, ${ }^{2}$ N Shepherd, ${ }^{3}$ T Cook ${ }^{11}$ Surgery, Gloucestershire Hospitals NHS Foundation Trust, Gloucester, UK; ${ }^{2}$ Biophotonics Research Unit, Gloucestershire Hospitals NHS Foundation Trust, Gloucester, UK; ${ }^{3}$ Pathology, Gloucestershire Hospitals NHS Foundation Trust, Gloucester, UK

Introduction The gold standard assessment of tissue biopsies from colonoscopy is histopathology. Infrared spectroscopy has potential to map biochemical changes across a tissue section distinguishing between different disease states. ${ }^{1}$ This may aid the pathologist or could lead to automated histopathological processing. This study attempted to determine biochemical changes in colorectal disease using infrared spectroscopy.

Methods Tissue biopsies were snap frozen at colonoscopy. 2-D spectral datasets were obtained from 10 micron thick sections of specimens, thawed to room temperature, using a Perkin Elmer infrared imaging system in transmission mode. Contiguous tissue sections stained with H\&E were reviewed by a senior consultant pathologist for comparison. Reference spectra from pure biochemicals (Sigma-Aldridge) were measured. Dot products ${ }^{2}$ of these reference spectra with the mapped spectral datasets were calculated to provide a correlation estimate represented as a pseudocolour image. Ordinary least squares analysis ${ }^{3}$ was also used to estimate the relative proportions of biochemical constituents from regions of interest.

Results Biochemical dot product correlation maps were obtained from normal and inflammatory tissue, hyperplastic polyps, adenomatous polyps and cancer. An example is shown in the accompanying figure. Distributions and proportions of DNA, glycogen, lipids and proteins were compared between areas of pathological interest (see figure 1).

Conclusion Infrared spectroscopy provides valuable biochemical information within colorectal pathologies. This information may aid the diagnosing pathologist and help develop automated histopathological processing.

Competing interests None.

Keywords biochemistry, colorectal carcinoma or dysplasia.
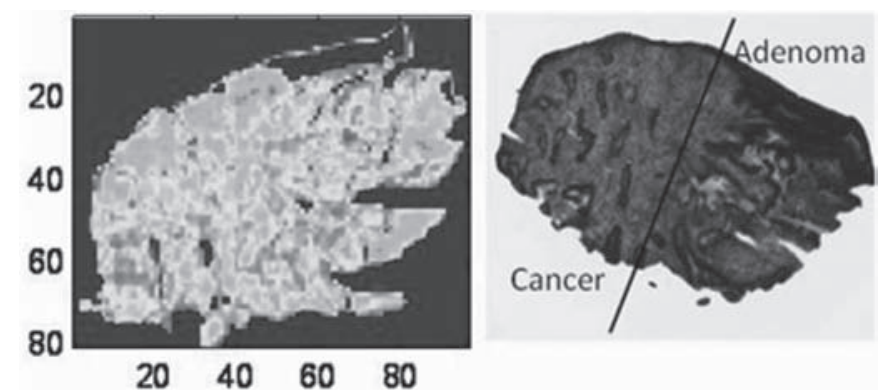

$20 \quad 40 \quad 60 \quad 80$

Pseudocolour map showing DNA content across a colon biopsy comprising mixed adenoma and cancer

\section{REFERENCES}

1. Lasch P, Haensch W, Naumann D, et al. Imaging colorectal adenocarcinoma using FTIR miscrospectroscopy and cluster analysis. Biochim Biophys Acta 2004;1688:176-86.

2. Isabelle M, Rogers K, Stone N. Correlation mapping: rapid method for identification of histological features and pathological classification in mid infrared spectroscopic images of lymph nodes. J Biomed Opt 2010 15(2):026030.

3. Shetty G, Kendall C, Sheperd N, et al. Raman spectroscopy: elucidation of biochemical changes in carcinogenesis of oesophagus. Br J Cancer 2006;94:1460-4.

Figure $1 \quad 0 \mathrm{C}-106$ 